\title{
Keine Integrierte Versorgung ohne eHealth - und umgekehrt
}

\section{Urs Zanoni ${ }^{a}$, Andre Rotzetter ${ }^{b}$}

${ }^{a} \mathrm{MPH}$, Departement Gesundheit und Soziales Kanton Aargau; ${ }^{\mathrm{b}}$ Präsidium Verein eHealth Aargau, Geschäftsführer Verein für Altersbetreuung im Oberen Fricktal

\begin{abstract}
Integrierte Versorgung und eHealth bedingen und inspirieren sich gegenseitig. Im Kanton Aargau werden die beiden Themen Hand in Hand entwickelt: Der Masterplan Integrierte Versorgung und das Programm eHealth Aargau sind die zentralen Bausteine der Vision «Vernetztes Gesundheitswesen Aargau».
\end{abstract}

\begin{abstract}
«Braucht es das wirklich?»: Als der Kanton Aargau im Herbst 2013 das Projekt Masterplan Integrierte Versorgung Aargau (MIVAG) vorstellte, war reichlich Skepsis zu vernehmen, namentlich bei Leistungserbringern. Die Frage drückte vor allem die Befürchtung aus, der Kanton wolle mehr staatliche Planung oder Regulierung verordnen.

Deshalb betonten die Verantwortlichen von Beginn weg die Philosophie des vierjährigen Projektes: Der MIVAG wird partnerschaftlich entwickelt und umgesetzt; er will auf dem Bestehenden aufbauen; er soll einen Handlungs- und Orientierungsrahmen schaffen und kein Planungs- oder Steuerungsinstrument sein. Und vor allem: Er ist Teil der Vision «Vernetztes Ge-
\end{abstract}

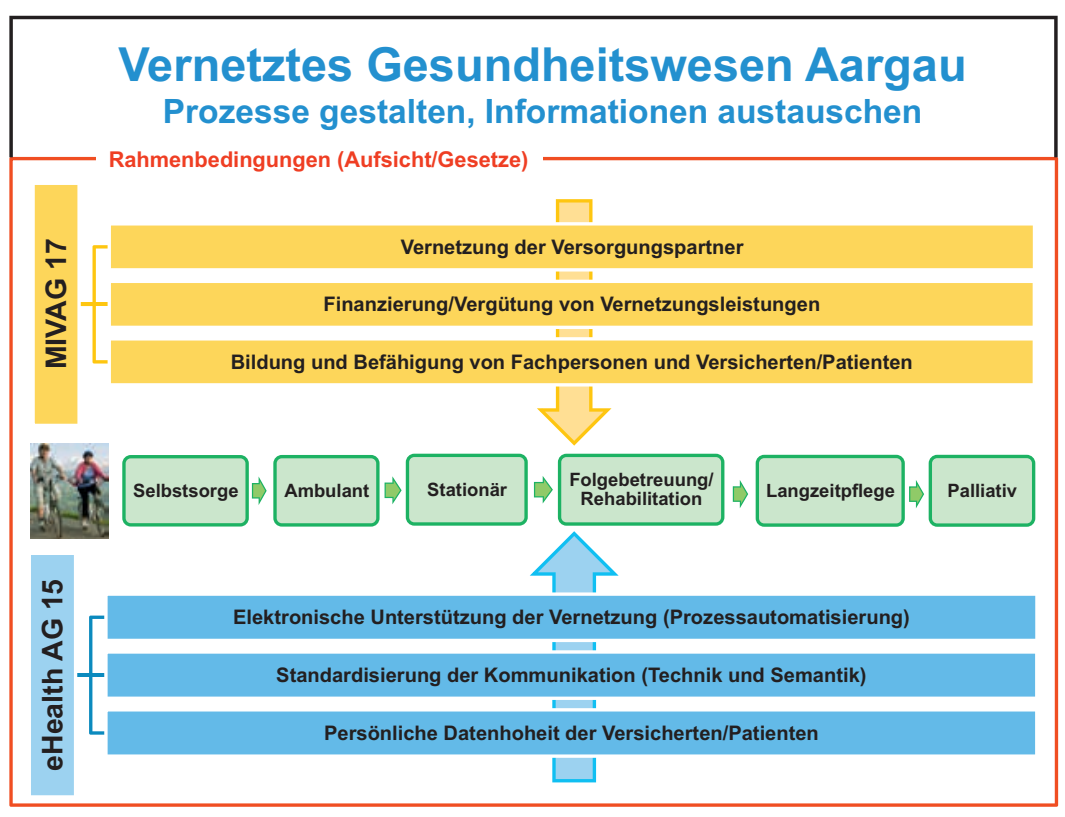

Abbildung 1: Grundlegend für das vernetzte Gesundheitswesen: physische Vernetzung von Menschen entlang des gesamten Behandlungspfades und digitale Unterstützung dieser Vernetzungsprozesse. sundheitswesen Aargau» mit dem Leitmotiv "Besser vernetzt - mehr Gesundheit für alle».

Die Vision wird auf zwei Wegen angestrebt (Abb.1): einerseits die physische Vernetzung von Menschen entlang des gesamten Behandlungspfades, andererseits die digitale Unterstützung dieser Vernetzungsprozesse. Für den einen Weg steht der MIVAG, für den anderen das Programm eHealth Aargau. Diese Symbiose von Integrierter Versorgung und eHealth ist ein Novum in der Schweiz. «Braucht es das wirklich?» Ja! Medizin, Pflege und Therapien haben sich in den letzten Jahrzehnten immer stärker differenziert, die Kontaktstellen zwischen den Fachpersonen vervielfacht. Die einzelnen Behandlungsschritte sind oft mangelhaft aufeinander abgestimmt, besonders wenn sie über Sektorengrenzen gehen: Die nächste Fachperson weiss häufig nicht oder nur lückenhaft, was die letzte oder vorletzte gemacht hat. Solche Informationsverluste führen zu Überdiagnostik, Mehrfachbehandlungen, Komplikationen, Notfall- und Wiedereinweisungen ins Spital - mit negativen Folgen für Behandlungsresultate, Patientensicherheit und Kostenentwicklung.

\section{Patientenperspektive als Verstärker}

Mit der besseren Vernetzung aller Partner der Gesundheits- und Krankheitsversorgung sollen diese negativen Folgen reduziert werden. Dazu gehört auch, vermehrt die Sicht der betroffenen Menschen zu berücksichtigen: Ihre Perspektive ist eine ergiebige Quelle, wenn die Behandlung so organisiert werden soll, dass die Lücken an den Übergängen möglichst klein sind und letztlich ein durchgehender Weg entsteht. Denn die Patienten sind die einzigen (allenfalls noch ihre Angehörigen oder andere Vertrauenspersonen), die den gesamten Behandlungs- und Betreuungsweg real erle- 
ben. Die Fachpersonen dagegen fokussieren (zwangsläufig) den einzelnen Behandlungsschritt und sehen häufig nur einen Teil des kranken Menschen: eine einzelne Diagnose, ein einzelnes Organ, eine einzelne Therapie.

Genauso wie die Patientensicht die Integration verstärken kann, verstärken sich Integrierte Versorgung und eHealth:

- Mehr physische Vernetzung zwischen den Leistungserbringern ist vor allem dann sinnvoll, wenn auch mehr behandlungsrelevante Daten, Bilder und Berichte elektronisch zur Verfügung stehen. Das bedeutet: keine Integrierte Versorgung ohne eHealth. Ein Beispiel dazu: Das Kantonsspital Aarau startete 2009 zusammen mit der Klinik Barmelweid und der aarReha Schinznach das Projekt OPTIMA zur Verbesserung der Aufnahme-, Behandlungs- und Übertrittsprozesse. Inzwischen sind eine Vielzahl an Instrumenten entwickelt worden, um die Prozesse elektronisch zu unterstützen. Zudem ist eine riesige Datenmenge zusammengekommen, die sich für die Versorgungsforschung nutzen lässt.

- Jede elektronische Vernetzung verlangt (auch) eine Überprüfung - und allfällige Anpassung - der physischen Behandlungs- und Betreuungsprozesse. Das bedeutet: ohne eHealth keine Integrierte Versorgung. Auch dazu ein Beispiel: Das Kantonsspital Baden entwickelte mit mehreren LeistungserbringerOrganisationen (Reha, Ärzte, Spitex, Pflegeheim) ein Set an Formularen für den elektronisch gestützten Spitaleintritt und -austritt. Gleichzeitig wurden die Prozesse analysiert und (neu) beschrieben.

Kommt hinzu: Die grossen Reformen im Gesundheitswesen geschehen nicht mehr auf Papier, sondern digital, Stichwort elektronisches Patientendossier. Und gleich wie die physische Vernetzung verlangt auch die Digitalisierung nach mehr und besserer Koordination; schliesslich gilt es, Hunderte von IT-Systemen auf einen gemeinsamen Nenner zu bringen, damit die Daten ungehindert fliessen.

\section{Guter Nährboden im Aargau}

Politische Grundlage für den MIVAG und das Programm eHealth Aargau ist die Gesundheitspolitische Gesamtplanung, die 2005 erstmals erarbeitet und 2010 aktualisiert wurde. In der Version 2025, die derzeit in der Anhörung ist und nächstes Jahr im kantonalen Parlament beraten wird, sind Integrierte Versorgung und eHealth in einer gemeinsamen Strategie zusammengefasst: «Alle Partner im Aargauer Gesund-

heitswesen (Leistungserbringer, Kostenträger, Versi-
cherte/Patienten) sind miteinander vernetzt und die Behandlungs- und Betreuungsprozesse integriert. Zur Gestaltung, Unterstützung und Vernetzung dieser Prozesse werden gezielt elektronische Informations- und Kommunikationstechnologien eingesetzt.ı*

Die Vernetzung im Kanton Aargau ist bereits weit fortgeschritten. Ein paar Beispiele: Es gibt das Versorgungsnetz Aargau Ost, das schon eine Vielzahl an Kooperationen etabliert hat. Argomed ist die grösste Managementgesellschaft für Ärztenetze in der Schweiz; im Aargau werden 43 Prozent der Versicherten in einem Ärztenetz betreut - der vierthöchste Wert im kantonalen Vergleich. Im September 2014 wurde der Verein eHealth Aargau gegründet, der schon über 200 Mitglieder aus allen Versorgungsbereichen zählt; der Verein wird ab Januar 2016 den Aufbau und Betrieb der (Stamm-)Gemeinschaft eHealth Aargau organisieren und der Bevölkerung das elektronische Patientendossier zugänglich machen. Zu guter Letzt hat der Kanton eine eGovernment- wie auch eine Hightech-Strategie, die Hand in Hand gehen mit der Digitalisierung im Gesundheitswesen.

Damit Integrierte Versorgung und eHealth dauerhaft zusammenfliessen, braucht es die Bereitschaft und den Einbezug aller Beteiligten:

- Die ambulanten und stationären Leistungserbringer sind seit Beginn dabei; damit ist auch die lokale und regionale Verankerung gewährleistet. Hinzu kommen weitere Versorgungspartner wie Fachorganisationen (für einzelne Krankheiten, Alter, Behinderung, Sucht), Gemeinden bzw. Regionalplanungsverbände, Versicherer, Bildungsinstitutionen.

- Die Aktivitäten gehen primär vom Bestehenden aus (Bottom-up-Ansatz): Die Leistungserbringer sind die Taktgeber; der Kanton wirkt als Koordinator, Moderator, Multiplikator und Dienstleister.

- Im Aargau besteht eine Vielzahl an zukunftsweisenden Projekten, die sich weiterentwickeln oder adaptieren lassen, zum Beispiel das neue Zuweiserportal der beiden Kantonsspitäler, das elektronische Rezept von Argomed und dem Apothekerverband, standardisierte Formulare für den Spitaleintritt und -austritt, die Optimierung der Übergänge zwischen Akutspitälern und Rehakliniken, standardisierte Übergabeprozesse von den Psychiatrischen Diensten zu Spitex-Organisationen.

- Der Kanton hat eine rechtliche Grundlage geschaffen, um automatisierte Abrufverfahren, wie sie für das elektronische Patientendossier nötig sind, in Pilotprojekten erproben zu können. Eine vergleichbare Pilotnorm ist für die Integrierte Versorgung vorgesehen, zum Beispiel für regionale, populationsorientierte Versorgungsmodelle. 
- Der Kanton steht zur Standardisierung der elektronischen Kommunikation (Technologie und Semantik), abgestimmt auf die eHealth-Strategie des Bundesrats und die Vorgaben von eHealth Suisse, dem Koordinationsorgan von Bund und Kantonen. Dadurch schafft er für die Leistungserbringer Rechtsund Investitionssicherheit.

- Der Kanton tauscht sich intensiv mit eHealth Suisse sowie dem Bundesamt für Gesundheit aus und beteiligt sich an nationalen Projekten wie Gesundheit 2020. Er bringt die Anliegen der aargauischen Versorgungspartner ein und stimmt sie mit den Vorgaben des Bundes ab.

- Die Projektpartner sind gut vernetzt mit der Politik; dadurch lassen sich inhaltliche Differenzen früh erkennen und diskutieren.

Korrespondenz:

Urs Zanoni, MPH

Leiter Masterplan Inte-

grierte Versorgung /

eHealth Aargau

Departement Gesundheit

und Soziales

Bachstrasse 15

CH-5000 Aarau

urs.zanoni[at]ag.ch
$-$

Patientensicht, Handlungskompetenz und Eigenverantwortung der Menschen werden im Aargau künftig Schlüsselbegriffe sein, denn die Gesundheitspolitische Gesamtplanung 2025 nennt neben Versorgungszielen auch Befähigungsziele - was für die Verbreitung des elektronischen Patientendossiers unerlässlich ist.

\section{Eine neue «Kultur des Gemeinsamen»}

Der Masterplan Integrierte Versorgung wie auch das Programm eHealth Aargau sind als Public Private Partnership konzipiert: Der Kanton finanziert den Anstoss, die Versorgungspartner investieren in Projekte. Das Modell gilt auch für den Aufbau und Betrieb der (Stamm-)Gemeinschaft im Aargau durch den Verein eHealth Aargau: Der Grosse Rat genehmigte dafür im Juni 2015 einen namhaften Kantonsbeitrag. Die Leistungserbringer investieren ein Mehrfaches davon, damit ihre IT-Systeme den Vorgaben von eHealth Suisse entsprechen und für das ePD nutzbar sind; hinzu kommen personelle Ressourcen, um die Systeme zu schulen und die (neuen) Arbeitsprozesse zu implementieren. Dass das verstärkte Zusammenspiel von Integrierter Versorgung und eHealth beträchtliches Potential aufweist, ist inzwischen unbestritten: Die Versorgung wird zeit- und ortsunabhängig; Medizin, Pflege und Therapie können vermehrt auch beim Patienten zu Hause stattfinden. Wenn mehr Fachpersonen mehr behandlungsrelevante Informationen im richtigen Moment zur Verfügung haben, können sich neue interprofessionelle Versorgungsmodelle etablieren. Und die Patienten (bzw. ihre Angehörigen oder sonstigen Vertrauenspersonen) werden vermehrt zu Partnern.

Andererseits bestehen auch beträchtliche Herausforderungen, allen voran: Wie lässt sich die Akzeptanz und Nutzung von elektronischen Hilfsmitteln verbessern, sowohl bei Fachpersonen wie auch bei Versicherten und Patienten? Und es braucht Modelle, um den (Mehr-)Aufwand für Koordination und Datenverarbeitung dauerhaft zu finanzieren.

Vor allem aber braucht es Menschen, die an das Zusammenwirken von Integrierter Versorgung und eHealth glauben. Und die sich kennen, verstehen und vertrauen. Weil die zentralen Herausforderungen der Gesundheits- und Krankheitsversorgung - Chronifizierung, Multimorbidität, Demenzen - eine neue «Kultur des Gemeinsamen» verlangen, bei der sich die Beteiligten auf Augenhöhe begegnen. 